\title{
A TOWER OF SPECTRA THAT REALIZES A CHAIN COMPLEX
}

\author{
PEDRO A. SUAREZ, S. J.
}

\begin{abstract}
This paper presents the construction of a tower of spectra $Y_{j}$ with $k$-invariants coming from the relations $\operatorname{Sq}^{\prime}\left(X \mathrm{Sq}^{2{ }^{\prime+1}}\right) \mathrm{Sq}^{\prime}\left(X \mathrm{Sq}^{2 \prime}\right)=0$ in $A / A \mathrm{Sq}^{1}$, for $0 \leqslant j \leqslant 5$ and $A=$ Steenrod algebra $\bmod 2$, such that $Y_{5}$ has prescribed homotopy groups: $\pi_{n}\left(Y_{5}\right)=Z$ (integers) if $n=2^{j+1}-2$, and zero otherwise.
\end{abstract}

I. Introduction. This paper presents an example of a tower of spectra associated with a chain complex over the Steenrod algebra module $A / A \mathrm{Sq}^{1}$ ( $A$ is the $\bmod 2$ Steenrod algebra).

A problem which is related to our present task was posed by F. Peterson in 1970 [2]. Peterson asks if there exists an $\Omega$-spectrum $\mathbf{X}(n)$ with homotopy groups

$$
\pi_{k}(\mathbf{X}(n))= \begin{cases}Z_{2} & \text { if } k=2^{r} n, r \geqslant 0 \\ 0 & \text { otherwise, }\end{cases}
$$

and such that the $r$ th stage Postnikov system has $k$-invariants coming from the relations

$$
\begin{aligned}
& \mathrm{Sq}^{n+1}=0, \\
& \mathrm{Sq}^{2 n+1} \mathrm{Sq}^{n+1}=0, \\
& \cdots \\
& \mathrm{Sq}^{2^{r-1} n+1}\left(\cdots \mathrm{Sq}^{n+1}\right)=0 .
\end{aligned}
$$

Furthermore, the 0th term of the spectrum should be a product of $K\left(Z_{2}, n\right) \times$ $K\left(Z_{2}, 2^{i} n\right) \times \cdots$ with twisted Hopf algebra structure, so that the homology generator in dimension $n$ generates a polynomial algebra.

By using an algebraic argument and the work of Segal [3], R. Steiner gives a positive answer to this question [4].

One can consider an extension of Peterson's problem and ask if there exists a spectrum (or a tower of spectra) that realizes a relation $a_{n+1} a_{n}=0$, where $a_{n}$ may be perhaps more complicated than $\mathrm{Sq}^{2 n+1} \mathrm{Sq}^{n+1}=0$. A simple case is the spectrum $b u$, whose tower realization is associated with the relation $\mathrm{Sq}^{3} \mathrm{Sq}^{3}=0$ in $A / A \mathrm{Sq}^{1}$. In

Received by the editors April 11, 1983

1980 Mathematics Subject Classification. Primary 55S10, 55S45.

Key words and phrases. Tower of spectra, chain complex, Steenrod algebra, fibrations of spectra, cohomology exact sequences. 
this paper we will construct a tower of spectra with $k$-invariants coming from the relation $\mathrm{Sq}^{1}\left(X \mathrm{Sq}^{2^{k+1}}\right) \mathrm{Sq}^{1}\left(X \mathrm{Sq}^{2^{\mathrm{k}}}\right)=0$ in $A / A \mathrm{Sq}^{1}$, where $X: A \rightarrow A$ is the canonical Thom antiautomorphism.

We will work in the category of spectra localized at two and homotopy classes of maps between such spectra. $K G(m)$ will represent the $m$-fold suspension of the Eilenberg-Mac Lane spectrum $K G$ (or $K G(0)$ ) for a group $G$. Thus, $K G(m)=$ $\sum^{m} K G(0)$, and $(K G(m))_{n}=K(G, m+n)$ for all nonnegative integers $m, n . b u$ is the $\Omega$-spectrum representing connective complex $K$-theory. The cohomology groups with coefficients in $Z_{2}$ of these spectra are well known [5].

II. Statement of results. First, we wish to state

Proposition 1. Let $D_{k}=A / A \mathrm{Sq}^{1}$ and $d_{k}=\operatorname{Sq}^{1}\left(X \mathrm{Sq}^{2^{k}}\right)$ for $k \geqslant 1$. Then

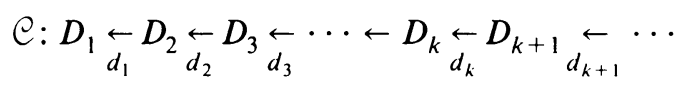

is a chain complex.

The proof of this proposition rests on

LEMma 2. The following relations hold in $A / A \mathrm{Sq}^{1}$ for $k \geqslant 1$
(a) $\operatorname{Sq}^{1}\left(X \mathrm{Sq}^{2^{k}}\right)=\operatorname{Sq}^{2^{k-1}} \operatorname{Sq}^{1}\left(X \mathrm{Sq}^{2^{k-1}}\right)$;
(b) $\left(X \mathrm{Sq}^{2^{k}}\right) \mathrm{Sq}^{1}\left(X \mathrm{Sq}^{2^{k+1}}\right)=0$;
(c) $\operatorname{Sq}^{1}\left(X \mathrm{Sq}^{2^{k+1}}\right) \mathrm{Sq}^{1}\left(X \mathrm{Sq}^{2^{k}}\right)=0$.

Finally, the main result is

THEOREM 3. The following tower of fibrations of spectra realizes a finite portion of $\bigodot$ $($ for $1 \leqslant k \leqslant 6)$ :

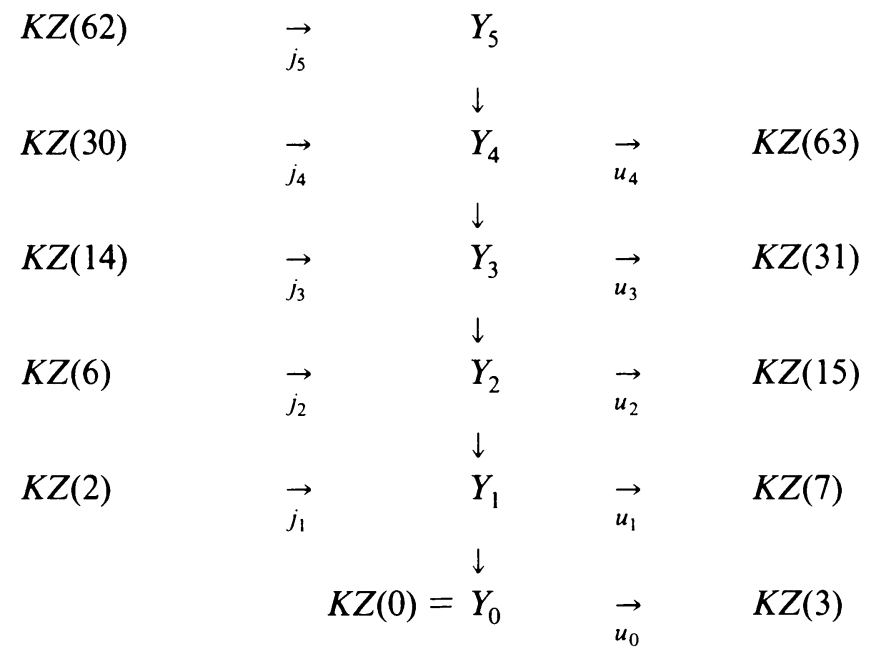


where

$$
\begin{aligned}
& u_{0}=b \operatorname{Sq}^{2}\left(i_{0}\right) \quad(b=\text { Bockstein homomorphism }), \\
& u_{1}=\operatorname{Sq}^{2}\left(v_{5}\right), \quad \text { with } v_{5}=\text { nonzero generator of } H^{5}\left(\Sigma^{5} b u ; Z\right), \\
& u_{2}=\operatorname{Sq}^{4} \operatorname{Sq}^{2} \operatorname{Sq}^{1}\left(v_{8}\right)=\left(X \operatorname{Sq}^{7}\right) v_{8} \text { and } v_{8} \in H^{8}\left(Y_{2} ; Z_{2}\right), \\
& u_{3}=\operatorname{Sq}^{8} \operatorname{Sq}^{4} \operatorname{Sq}^{1}\left(v_{18}\right)=\left(X \operatorname{Sq}^{13}\right) v_{18} \text { and } v_{18} \in H^{18}\left(Y_{3} ; Z_{2}\right), \\
& u_{4}=\operatorname{Sq}^{16} \operatorname{Sq}^{8} \operatorname{Sq}^{1}\left(v_{38}\right) \text { and } v_{38} \in H^{38}\left(Y_{4} ; Z_{2}\right) .
\end{aligned}
$$

Furthermore, the homotopy groups of the spectrum $Y_{5}$ are

$$
\pi_{i}\left(Y_{5}\right)= \begin{cases}Z & \text { if } i=2^{k}-2,1 \leqslant k \leqslant 6 \\ 0 & \text { otherwise. }\end{cases}
$$

III. Proofs of 1,2 and 3. The proof of Proposition 1 clearly rests on the assertion of Lemma 2(c). Lemma 2(a) is proved by mathematical induction and the use of the relation

$$
X\left(\mathrm{Sq}^{2^{n}-k}\right)=\mathrm{Sq}^{2^{n-1}} \mathrm{Sq}^{2^{n-2}} \cdots \mathrm{Sq}^{2^{k-1}}\left(X \mathrm{Sq}^{2^{k-1}-k}\right)
$$

for any integers $n, k$ such that $n \geqslant k \geqslant 1$ (see [1]). 2(b) is proved by using 2(a) and the Adem relation $\mathrm{Sq}^{2 n-1} \mathrm{Sq}^{n}=0.2$ (c) follows immediately from (b).

The proof of Theorem 3 involves the construction of a Postnikov tower (the $T$-tower) and then an induced tower of spectra $Y_{k}(1 \leqslant k \leqslant 6)$. The final picture looks like this:

$\begin{array}{lcccccc}K Z(62) & \overrightarrow{j_{5}} & Y_{5} & \overrightarrow{a_{5}} & T_{5} & & \\ & & \downarrow & & \downarrow & & \\ K Z(30) & \overrightarrow{j_{4}} & Y_{4} & \overrightarrow{a_{4}} & T_{4} & \overrightarrow{f_{4}} & K Z(63) \\ & & \downarrow & & \downarrow & & \\ K Z(14) & \overrightarrow{j_{3}} & Y_{3} & \overrightarrow{a_{3}} & T_{3} & \overrightarrow{f_{3}} & K Z(31) \\ & & \downarrow & & \downarrow & & \\ K Z(6) & \overrightarrow{j_{2}} & Y_{2} & \overrightarrow{a_{2}} & T_{2} & \overrightarrow{f_{2}} & K Z(15) \\ & \overrightarrow{j_{1}} & Y_{1} & \overrightarrow{a_{1}} & T_{1} & \overrightarrow{f_{1}} & K Z(7) \\ K Z(2) & \overrightarrow{j_{1}} & \downarrow & & \downarrow & & \\ & \overrightarrow{j_{0}=\mathrm{Id}} & Y_{0} & \overrightarrow{a_{0}} & \Sigma^{3} b u=T_{0} & \overrightarrow{f_{0}} & K Z(3) \\ & & & & & \end{array}$

The following is a sketch of the construction of this double tower of spectra. A more detailed discussion can be found in [6].

1. $f_{0} \in H^{3}\left(\Sigma^{3} b u ; Z\right)=\left[\Sigma^{3} b u, K Z(3)\right]$ corresponds to the generator 1 . 
2. Each $T_{i+1}$ is induced by $f_{i}$ over the path fibration of the Eilenberg-Mac Lane spectrum $K Z\left(2^{i+2}-1\right)$ for $0 \leqslant i \leqslant 4$.

3. $a_{0} \in\left[K Z(0), \Sigma^{3} b u\right]$ is such that $f_{0} a_{0}=b \mathrm{Sq}^{2}\left(i_{0}\right) \in[K Z(0), K Z(3)]$, where $b$ is the Bockstein homomorphism in cohomology and $i_{0}$ is the bottom class in $H^{0}(K Z(0) ; Z)$.

4. $T_{1} \simeq \Sigma^{5} b u$. This can be seen by considering the sequence of fibrations $\sum^{n+3} b u$ $\rightarrow \Sigma^{n+1} b u \rightarrow K Z(n+1)$, obtained by Bott periodicity.

5. The $T$-tower is constructed as follows:

$$
\begin{aligned}
& f_{0}=\text { nonzero generator of } H^{3}\left(\Sigma^{3} b u ; Z\right), \\
& f_{1}=\operatorname{Sq}^{2}\left(v_{5}\right) \in H^{7}\left(\Sigma^{5} b u ; Z\right), \quad \text { where } v_{5}=\text { nonzero generator of } H^{5}\left(\Sigma^{5} b u ; Z\right), \\
& f_{2}=\operatorname{Sq}^{4} \operatorname{Sq}^{2} \operatorname{Sq}^{1}\left(v_{8}\right) \in H^{15}\left(T_{2} ; Z\right), \\
& f_{3}=\operatorname{Sq}^{8} \operatorname{Sq}^{4} \operatorname{Sq}^{1}\left(v_{18}\right) \in H^{31}\left(T_{3} ; Z\right), \\
& f_{4}=\operatorname{Sq}^{16} \operatorname{Sq}^{8} \operatorname{Sq}^{1}\left(v_{38}\right) \in H^{63}\left(T_{4} ; Z\right),
\end{aligned}
$$

where each $v_{m}$ appears by exactness of the long exact sequence in cohomology of each fibration $\left(m=2^{k+1}+2^{k-1}-2 ; k=2,3,4\right)$ and each $f_{k}$ is an integer class $(0 \leqslant k \leqslant 4)$.

6. The $Y$-tower is induced over the $T$-tower in the following way. At the first stage we have

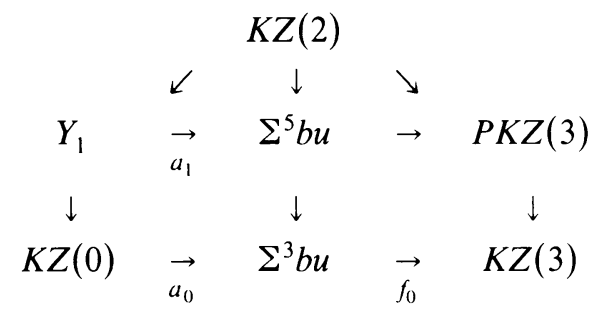

where $a_{0} \in\left[K Z(0), \Sigma^{3} b u\right]$ such that

$$
f_{0} a_{0}=b \mathrm{Sq}^{2}\left(i_{0}\right) \in[K Z(0), K Z(3)] \cong H^{3}(K Z(0) ; Z)
$$

Then $Y_{1}$ is the total spectrum over the path fibration $K Z(2) \hookrightarrow P K Z(3) \rightarrow K Z(3)$ induced by $f_{0} a_{0}$. By the functorial properties of induced fibrations, $Y_{1}$ is equivalent to the total spectrum induced by $a_{0}$ over the fibration $K Z(2) \hookrightarrow \Sigma^{5} b u \rightarrow \Sigma^{3} b u$, so there exists a map $a_{1}: Y_{1} \rightarrow \Sigma^{5} b u$.

Taking the composition of $a_{1}$ with $f_{1} \in\left[T_{1}, K Z(7)\right]$ we obtain a map $f_{1} a_{1} \in$ $\left[Y_{1}, K Z(7)\right]$ which induces the spectrum $Y_{2}$ over the path fibration $K Z(6) \hookrightarrow P K Z(7)$ $\rightarrow K Z(7)$, and the process can be repeated again, obtaining $u_{k}=f_{k} a_{k}(0 \leqslant k \leqslant 4)$.

7. The following diagram illustrates how cohomology classes are mapped at each level of the tower in their respective long exact cohomology sequences (the rings on the right indicate the coefficients in cohomology and $\tau$ is transgression): 


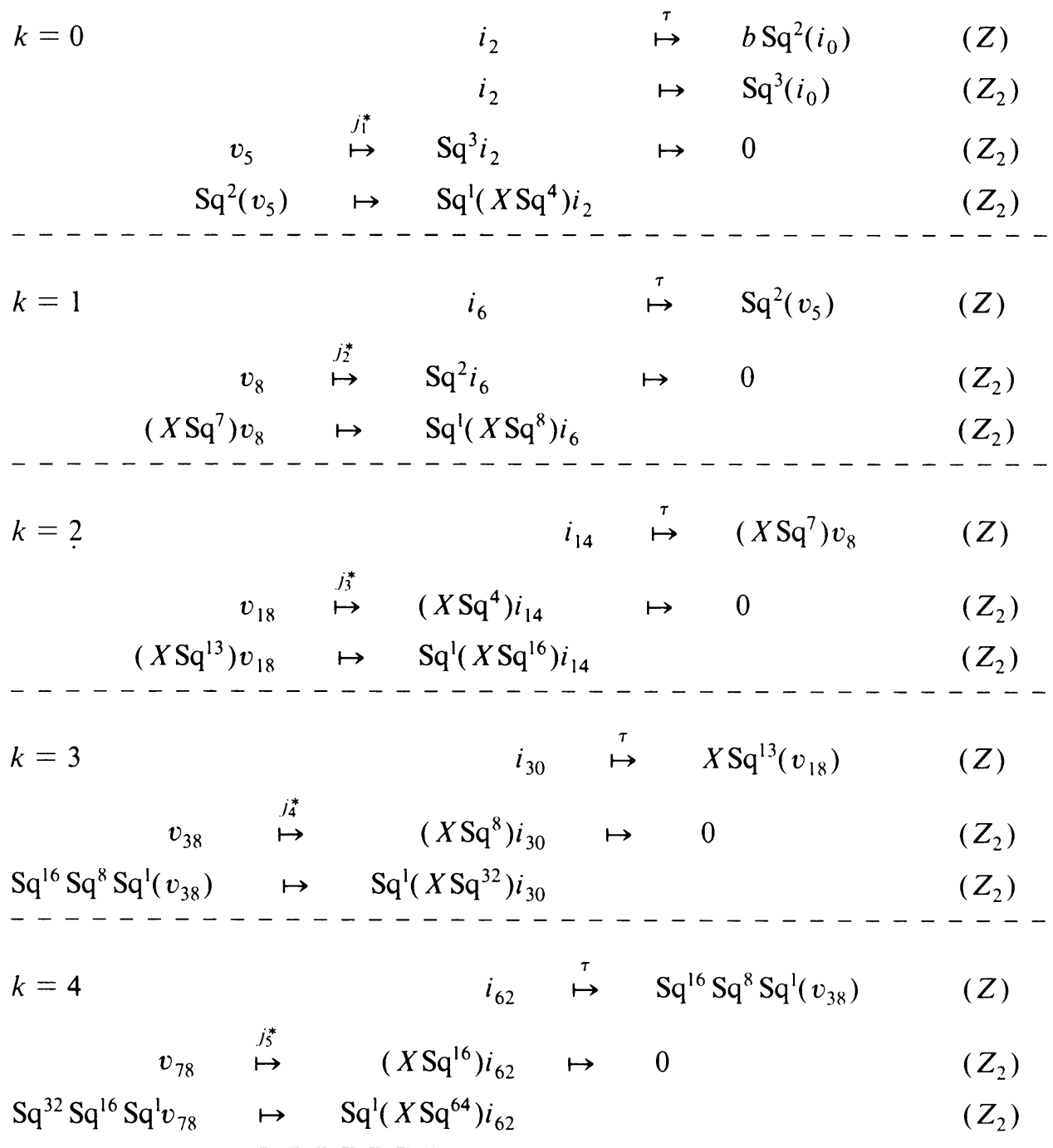

8. It remains to show that this tower realizes (or is associated with) the chain complex $\mathrm{C}$. By Lemma 2,

$$
d_{k} d_{k+1}=\operatorname{Sq}^{1}\left(X \mathrm{Sq}^{2^{k+1}}\right) \mathrm{Sq}^{1}\left(X \mathrm{Sq}^{2^{k}}\right)=0 \bmod A / A \mathrm{Sq}^{1}
$$

for all nonnegative integers $k$. But we also have for $1 \leqslant k \leqslant 5$ :

$$
0=\operatorname{Sq}^{l} X \operatorname{Sq}^{2^{k+1}} \operatorname{Sq}^{l} X \operatorname{Sq}^{2^{k}}\left(i_{2^{k}-2}\right)=\operatorname{Sq}^{l} X \operatorname{Sq}^{2^{k+1}} j_{k-1}^{*}\left(\operatorname{Sq}^{2^{k-1}} \operatorname{Sq}^{2^{k-2}} \operatorname{Sq}^{1}\left(v_{s}\right)\right) \text {, }
$$

letting $s=2^{k}+2^{k-2}-2$. We note that

$$
\mathrm{Sq}^{2^{k-1}} \mathrm{Sq}^{2^{h-2}} \mathrm{Sq}^{1}\left(v_{s}\right)=\tau\left(i_{2^{h+1}-2}\right)
$$

Thus, we obtain

$$
0=\mathrm{Sq}^{1} X \mathrm{Sq}^{2^{k+1}} j_{k-1}^{*} \tau\left(i_{2^{k+1}-2}\right)=j_{k-1}^{*} \tau \mathrm{Sq}^{1} X \mathrm{Sq}^{2^{k+1}}\left(i_{2^{k+1}-2}\right)
$$


The homotopy groups of the spectrum $Y_{5}$ are those stated in the theorem, as can be easily checked from the diagram, by the exact homotopy sequence at each stage.

Technical difficulties with this method preclude our going higher and obtaining an infinite tower. Concretely, it is not clear that a class $v_{s} \in H^{t}\left(Y_{k} ; Z_{2}\right)$ exists $(s=$ $\left.2^{k+1}+2^{k-1}-2 ; t=2^{k+2}-1\right)$ such that $\mathrm{Sq}^{2^{k}} \mathrm{Sq}^{2^{k-1}} \mathrm{Sq}^{1}\left(v_{s}\right)$ is an integer class for every $k \geqslant 5$.

\section{REFERENCES}

1. D. M. Davis, The antiautomorphism of the Steenrod algebra, Proc. Amer. Math. Soc. 44 (1974), 235-236.

2. R. J. Milgram (Editor), Problems presented to the 1970 Amer. Math. Soc. Summer Colloquium in Algebraic Topology, Algebraic Topology, Proc. Sympos. Pure Math., vol. 22, Amer. Math. Soc., Providence, R. I., 1971, Problem 43, p. 194.

3. G. Segal, The multiplicative group of classical cohomology, Quart. J. Math. Oxford Ser. (2) 26 (1975), 289-293.

4. R. Steiner, Decompositions of groups of units in ordinary cohomology, Quart. J. Math. Oxford Ser. (2) 30 (1979), 483-494.

5. R. E. Stong, Determination of $H^{*}\left(B O(k, \ldots, \infty) ; Z_{2}\right)$ and $H^{*}\left(B U(k, \ldots, \infty) ; Z_{2}\right)$, Trans. Amer. Math. Soc. 107 (1963), 526-544.

6. P. A. Suarez, A spectrum realization of a finite chain complex over the cohomology ring of the stable integral Eilenberg-Mac Lane space at the prime two, Thesis, Northwestern Univ., Evanston, Ill., 1977.

Department of Mathematics, Fordham University, BronX, New York, 10458

Current address: Departamento de Matemáticas, Universidad Autónoma de Santo Domingo, Santo Domingo, Dominican Republic 